\title{
I CANDIDATI. VISIONI POLITICHE \\ E CARRIERE
}

\author{
di Liborio Mattina e Alessandro Tonarelli
}

L'analisi delle candidature è suscettibile di diverse letture. In questo saggio ne privilegeremo due. La prima, prevalente, individuerà le caratteristiche dell'offerta di rappresentanza, la seconda le modalità di selezione del ceto politico parlamentare. L'una ci spiega la «proposta» che partiti e schieramenti elaborano per catturare il voto dell'elettore suggerendogli di essere rappresentato all'interno delle loro fila meglio che in quelle dei concorrenti. L'altra esamina il processo, più interno ai partiti, attraverso il quale avviene la riproduzione del ceto dirigente partitico e parlamentare.

Nel nostro saggio manterremo distinta l'analisi delle due questioni esaminando l'offerta di rappresentanza con riferimento alle attività professionali dei candidati, e i percorsi di formazione del nuovo ceto con una ricognizione sulle esperienze politiche dei candidati precedenti la loro designazione. I risultati della ricerca verranno presentati dedicando particolare attenzione all'analisi su base sovraregionale in considerazione della rilevanza che la segmentazione territoriale del mercato elettorale italiano ha assunto nella elaborazione delle strategie elettorali dei partiti.

\section{I candidati}

La nostra indagine esamina $\mathrm{i}$ candidati in tutti $\mathrm{i}$ collegi uninominali per l'elezione della Camera e del Senato presentati dai tre principali schieramenti concorrenti alle elezioni parlamentari del 21 aprile: la coalizione Ulivo più Partito della rifondazione comunista, il Polo per le libertà e la Lega Nord. La rilevazione ha coperto l'intero territorio nazionale per un totale dei candidati considerati pari a 1.763 unità $^{1}$. Essi costituiscono il $60 \%$

1 Desideriamo ringraziare i seguenti amici e colleghi che hanno collaborato alla rac-

RIVISTA ITALIANA DI SCIENZA POLITICA / a. XXVI, n. 3, dicembre 1996 
TAB. 1. Il numero dei candidati distinto per schieramento e area geografica

\begin{tabular}{|c|c|c|c|c|c|c|c|}
\hline \multirow[t]{2}{*}{ Area } & \multicolumn{3}{|c|}{ Schieramento } & \multicolumn{2}{|c|}{ Ramo del Parlamento } & \multicolumn{2}{|c|}{ Totale } \\
\hline & Ulivo + Rc & Polo & $\begin{array}{l}\text { Lega } \\
\text { nord }\end{array}$ & Camera & Senato & N. & $\%$ \\
\hline Nord & 267 & 266 & 263 & 536 & 260 & 796 & 45,1 \\
\hline Centro & 120 & 120 & 93 & 213 & 120 & 333 & 18,9 \\
\hline Sud & 317 & 317 & - & 427 & 207 & 634 & 36 \\
\hline Totale & 704 & 703 & 356 & 1.176 & 587 & 1.763 & 100 \\
\hline
\end{tabular}

NB: Il Centro comprende l'Emilia Romagna ed esclude il Lazio che viene ricompreso nel Sud di cui fanno parte anche Sicilia e Sardegna.

del totale dei candidati che si sono affrontati nei collegi uninominali e da essi è scaturito il 98,7\% degli eletti con il sistema maggioritario. Il nostro campo d'indagine coincide, in altri termini, con l'universo di tutti i candidati rilevanti. La loro distribuzione per schieramento, area geografica e ramo parlamentare è esposta nella tabella 1 .

I dati acquisiti riguardano l'età, il reddito, lo schieramento e il partito di appartenenza, la professione, gli eventuali precedenti parlamentari, le eventuali cariche ricoperte in partiti, organizzazioni di rappresentanza di interessi economici e non, le eventuali esperienze nelle amministrazioni locali. Le informazioni sono state attinte da quattro tipi di fonti: i profili biografici pubblicati nel corso della campagna elettorale sulle pagine locali dei quotidiani; i dati forniti dalle segreterie nazionali e regionali dei principali partiti; l'«Elenco dei deputati e dei senatori della XII legislatura»; la banca dati sulle elezioni del 1994 costituita presso il Dipartimento di Scienza della politica e Sociologia politica dell'Università di Firenze. Non sempre è stato possibile rilevare tutte le informazioni ricercate; per tale motivo abbiamo deciso di far riferimento nel corso dell'analisi, in alcune occasioni, ai candidati degli otto maggiori partiti (pari al 96,5\% dell'intero universo).

colta del materiale empirico: Nuccio Amato, Giovanni Civita, Bartolo Costanzo, Giuseppe Gangemi, Guido Legnante, Emanuele Raco, Gianni Riccamboni, Carlo Riviello, Giacomo Sani, Lorenza Sebesta, Mauro Tebaldi. Un sincero ringraziamento anche a quanti, nei giornali e nei partiti, ci hanno aiutato a raccogliere i dati: Maurizio Chiocchetti, Antonio Del Giudice, Cesare Di Gesaro, Carla Errico, Livia Ferro, Luigia Ierace, Concetto Iozzia, Emma Luxardo, Corrado Maiorca, Giacomo Mameli, Giuseppe Mazzone, Daniela Pagnotti, Michele Partipilo, Federico Reviglio, Gianfranco Salmoiraghi. 


\section{Caratteri generali dei candidati}

I candidati alle elezioni del 21 aprile erano nella stragrande maggioranza $(91,8 \%)$ uomini, con un reddito medio elevato e con un'età media di 46 anni per la Camera e di 53 per il Senato. Questo profilo generale del candidato 1996 non è immediatamente decifrabile se confrontato con quello dei candidati alle politiche del $1994^{2}$ perché, a parte la conferma dell'elevato reddito $^{3}$, propone, a livello aggregato, candidati leggermente più giovani ${ }^{4}$ ma tra le cui fila appare ulteriormente ridotto lo spazio già modesto offerto in passato alle donne. L'abbassamento dell'età indicherebbe una maggiore apertura dei canali della rappresentanza che, però, la riduzione del numero delle donne smentisce.

L'apparente contraddittorietà dei dati viene superata dall'analisi disaggregata delle fasce d'età in cui sono collocabili i candidati (tab. 2). Da essa emerge che $i$ candidati più giovani erano quelli della Lega nord, e tra essi le donne erano più numerose della media $(9,8 \%)$. In particolare, quasi la metà $(48,5 \%)$ dei candidati leghisti aveva meno di 46 anni. Al contrario, i candidati di tutti gli altri partiti risultano più spostati, rispetto ai dati del 1994, sulle fasce d'età centrali o anziane. Notevole, a questo riguardo, lo spostamento effettuato dai candidati del Pds che hanno ridotto del $10,5 \%$ la loro presenza nelle due fasce d'età più giovani (dal $41,6 \%$ al $31,1 \%$ ).

$\mathrm{Nel}$ complesso, i caratteri generali dei candidati indicano una tendenza riscontrabile in tutti i partiti, con l'eccezione della Lega, a presentare un'offerta di rappresentanza concentrata in segmenti sociali più specializzati, cioè a puntare su un personale politico più esperto di quello presentato nel 1994. Da tale tendenza, che verrà confermata da ulteriori riscontri empirici in questo contributo e in quello sugli eletti (Verzichelli 1996), si è scostata la Lega perché interessata a valorizzare elementi meno esperti ma più fedeli, cioè più militanti e radicati sul territorio.

2 I riscontri vanno considerati con cautela perché risultano dal confronto tra i dati desunti da un campione (Mattina 1995, 236-238) e quelli, ora utilizzati, relativi all'universo dei casi.

3 I nostri dati sul reddito riguardano appena 455 candidati. I più facoltosi, come nel 1994, erano i candidati del Polo (ił 55,5\% guadagnava più di 100 milioni di reddito annuo), seguiti da vicino dall'Ulivo più Prc, $(42,8 \%$ ) e a molta distanza dai candidati leghisti (solo il $27,6 \%$ di essi dichiarava redditi superiori ai 100 milioni).

4 Cfr. Mattina (1995), e anche Censis (1994). 
TAB. 2. Le fasce d'età per partito (\%)

\begin{tabular}{lrrrrrrrrr}
\hline & Prc & Pds & Ppi & $\begin{array}{c}\text { Lista } \\
\text { Dini }\end{array}$ & $\begin{array}{r}\text { Lega } \\
\text { nord }\end{array}$ & $\begin{array}{l}\text { Ccd- } \\
\text { Cdu }\end{array}$ & $\begin{array}{l}\text { Forza } \\
\text { Italia }\end{array}$ & An & Totale \\
\hline $27-35$ anni & - & 2,4 & 3,5 & 1,7 & 19,4 & 6,6 & 7,2 & 7,7 & 8,0 \\
$36-45$ & 34,1 & 28,7 & 22,4 & 28,3 & 29,2 & 31,1 & 30,9 & 24,5 & 28,4 \\
$46-55$ & 43,2 & 47,3 & 40,8 & 41,7 & 38,8 & 38,7 & 37,8 & 35,6 & 40,6 \\
$56-65$ & 15,9 & 17,5 & 27,0 & 23,3 & 10,4 & 19,8 & 19,5 & 24,5 & 18,5 \\
Oltre 65 & 6,8 & 4,1 & 6,3 & 5 & 2,2 & 3,8 & 4,6 & 7,7 & 4,6 \\
& & & & & & & & & \\
Totale & 100 & 100 & 100 & 100 & 100 & 100 & 100 & 100 & 100 \\
(N.) & $(44)$ & $(338)$ & $(174)$ & $(60)$ & $(356)$ & $(106)$ & $(307)$ & $(208)$ & $(1.593)$ \\
\hline
\end{tabular}

\section{La territorializzazione mancata}

Rimane da chiarire se i partiti abbiano cercato di favorire, o non scoraggiare, il radicamento territoriale dei loro candidati. Il quesito ha una sua rilevanza perché è opinione diffusa che la territorializzazione e il decentramento dei processi di selezione dei candidati vengano favoriti dall'esistenza di sistemi elettorali maggioritari uninominali. L'adozione di tali sistemi potrebbe, perciò, produrre la riduzione del numero di politici di professione tra i candidati e i parlamentari a vantaggio di altre categorie. Qui ci limitiamo ad affrontare in modo indiretto l'argomento, per controllare se - attraverso una certa territorializzazione dei candidati - l'offerta di rappresentanza abbia raggiunto una maggiore sintonia con la società civile delle singole constituencies.

L'assunto di partenza è che si registra un elevato grado di territorializzazione delle candidature quando si constata la riconferma generalizzata dei parlamentari uscenti e/o già presentati in elezioni precedenti, e quando tale riconferma avviene con la collocazione dei candidati parlamentari nei medesimi collegi $o$ in collegi che insistono negli stessi ambiti territoriali delle precedenti consultazioni elettorali. In termini operativi abbiamo cercato di misurare l'incidenza dei parlamentari uscenti con i dati presentati nella tabella 3 . Essi mostrano che il $36,2 \%$ dei candidati era costituito da parlamentari uscenti. Ma, aggiungiamo, costoro sono riusciti a conquistare circa il 54\% dei seggi parlamentari disponibili nella quota maggioritaria. Ciò potrebbe far ritenere che l'elevato tasso di successo elettorale dei parlamentari uscenti sia imputabile alla loro maggiore disponibilità di risorse politiche tra le quali possiamo annoverare il radicamento territoriale. 
TAB. 3. Incidenza di parlamentari uscenti sul totale dei candidati di ciascun partito (\%)

\begin{tabular}{lccccccccc}
\hline & Prc & Pds & Ppi & $\begin{array}{r}\text { Lista } \\
\text { Dini }\end{array}$ & $\begin{array}{r}\text { Lega } \\
\text { nord }\end{array}$ & $\begin{array}{r}\text { Ccd- } \\
\text { Cdu }\end{array}$ & $\begin{array}{r}\text { Forza } \\
\text { Italia }\end{array}$ & An & Totale \\
\hline $\begin{array}{l}\text { Candidati } \\
\text { parlamentari } \\
\text { uscenti }\end{array}$ & 56,8 & 49,7 & 24,7 & 23,8 & 25,4 & 33,9 & 34,3 & 47,7 & 36,2 \\
$\begin{array}{l}\text { Candidati } \\
\text { non } \\
\text { parlamentari }\end{array}$ & 43,2 & 50,3 & 75,3 & 76,2 & 74,6 & 66,1 & 65,7 & 52,3 & 63,8 \\
$\begin{array}{l}\text { Totale } \\
(N .)\end{array}$ & 100 & 100 & 100 & 100 & 100 & 100 & 100 & 100 & 100 \\
\hline & $(44)$ & $(340)$ & $(182)$ & $(63)$ & $(356)$ & $(127)$ & $(338)$ & $(237)$ & $(1.687)$ \\
\hline
\end{tabular}

In realtà, l'analisi disaggregata dei parlamentari uscenti per appartenenza partitica suggerisce che la loro incidenza sul totale dei candidati sia da attribuire meno al loro grado di territorializzazione e molto più alle vicende interne ai partiti nei mesi precedenti le elezioni, e alle scelte delle direzioni nazionali. La tabella 3 consente, infatti, di ripartire i partiti politici in tre diversi gruppi, in corrispondenza di differenti quote di candidati parlamentari uscenti, ciascuna risultante da decisioni e iniziative adottate a livello centrale. La quota più alta, vicina al $50 \%$, è relativa ai candidati parlamentari uscenti appartenenti al Prc, Pds e ad An, cioè ai tre partiti più strutturati e centralizzati che hanno espressamente mostrato grande attenzione alla riproduzione del proprio ceto politico. In un secondo gruppo di partiti (Forza Italia e Ccd-Cdu) registriamo un'incidenza dei parlamentari uscenti sui candidati superiore al $30 \%$. Si tratta, in questo caso, dei partiti che alla vigilia delle elezioni hanno composto le loro liste elettorali fàcendo posto anche ai candidati provenienti da altre formazioni. Il terzo gruppo, per il quale registriamo un'incidenza degli uscenti attorno al $25 \%$ sui candidati, è composto da quelle formazioni che, per ragioni diverse, sono state interessate da profondi cambiamenti nei mesi e nelle settimane precedenti le elezioni: la lista Rinnovamento nata a ridosso delle elezioni; il Ppi reduce da una grave scissione interna; la Lega mobilitata, attraverso consultazioni primarie, a ridare potere alla base nella selezione dei candidati. Un'ulteriore conferma della scarsa territorializzazione dei candidati è data dall'elevato grado di ricambio delle candidature $(56,1 \%)$ al quale si è accompagnata una certa mobilità tra $\mathrm{i}$ collegi che ha interessato sia gli uscenti rieletti (Di Virgilio 1996, tab. 15) che i 
TAB. 4. Ricandidature per area geografica e schieramento (\%)

\begin{tabular}{|c|c|c|c|c|c|c|c|c|}
\hline & \multicolumn{2}{|c|}{ Nord } & \multicolumn{2}{|c|}{ Centro } & \multicolumn{2}{|c|}{ Sud } & \multicolumn{2}{|c|}{ Italia } \\
\hline & $\begin{array}{c}\text { Nuove } \\
\text { candidature }\end{array}$ & $\begin{array}{l}\text { Ricandidati } \\
\text { dal } 1994\end{array}$ & $\begin{array}{c}\text { Nuove } \\
\text { candidature }\end{array}$ & $\begin{array}{l}\text { Ricandidati } \\
\text { dal } 1994\end{array}$ & $\begin{array}{c}\text { Nuove } \\
\text { candidature }\end{array}$ & $\begin{array}{c}\text { Ricandidati } \\
\text { dal } 1994\end{array}$ & $\begin{array}{c}\text { Nuove } \\
\text { candidature }\end{array}$ & $\begin{array}{l}\text { Ricandidati } \\
\text { dal } 1994\end{array}$ \\
\hline Ulivo + Rc & 70 & 30 & 20 & 80 & 47 & 53 & 51,1 & 48,9 \\
\hline Polo & 53 & 47 & 82,5 & 17,5 & 41 & 59 & 52,6 & 47,4 \\
\hline Lega nord & 65,4 & 34,6 & 93,5 & 6,5 & - & - & 72,8 & 27,2 \\
\hline Totale & 62,8 & 37,2 & 63,1 & 36,9 & 44 & 56 & 56,1 & 43,9 \\
\hline
\end{tabular}

candidati vecchi e nuovi (tab. 4). Entrambi questi fenomeni sono stati provocati dalla decisione delle direzioni nazionali dei partiti di modulare l'offerta in funzione delle caratteristiche del mercato elettorale. È accaduto, cioè, che le leadership partitiche, rese consapevoli dai risultati delle elezioni del 1994 della scarsa competitività elettorale di due delle tre maggiori aree geo-politiche del paese, abbiano deciso di presentare candidature nuove soprattutto nei collegi difficilmente espugnabili (per l'Ulivo il Nord; per il Polo il Centro); hanno, invece, confermato nei collegi delle aree per loro sicure i candidati parlamentari uscenti («squadra che vince non si cambia!») e i nuovi che intendevano sostenere (tab. 4, ma vedi anche il commento alla tab. 7). Nel Sud, a differenza che nelle altre due aree, la maggiore incertezza della competizione elettorale ha prodotto - nei due schieramenti maggiori - un minor ricambio delle candidature e una maggiore riconferma di molti candidati non eletti nel 1994 per uno scarto limitato di voti (Bartolini e D'Alimonte 1995 b, tabb. 12 e 13 ).

La modulazione centralizzata dell'offerta in relazione al grado di competitività delle diverse aree del mercato elettorale è stata anche adottata per far fronte alla comprensibile indisponibilità a ricandidarsi di candidati frustrati dall'esito negativo della precedente competizione elettorale. I dati della tabella 4 suggeriscono che l'aspettativa di una nuova sconfitta può avere provocato una vera e propria fuga di candidati dell'Ulivo dal Nord e di quelli del Polo dal Centro. Da ciò la necessità dei leaders partitici di scegliere volti nuovi. L'elevatissimo tasso di ricambio tra i candidati della Lega è, al contrario che per l'Ulivo e il Polo, il risultato di una precisa scelta politica finalizzata a valorizzare la componente movimentista dell'organizzazione che, nei piani del leader, avrebbe rincuorato i seguaci turbati 
dalle defezioni di parlamentari leghisti e assicurato l'emergenza di una leva di candidati, ed eventuali parlamentari, fedeli al partito e devoti al capo.

\section{L'offerta di rappresentanza}

Le candidature proposte nei collegi maggioritari (tab. 5) presentano, nella sostanza, le medesime caratteristiche sociologiche rilevate nel 1994 (Mattina 1995). I partiti politici hanno proposto candidati provenienti in primo luogo dalle professioni cosiddette predisposte, liberi professionisti, docenti universitari e insegnanti ${ }^{5}$, cioè da quei ceti sociali più inclini ad accettare l'invito a partecipare alla competizione elettorale perché hanno più possibilità di successo. I partiti hanno, inoltre, fatto ricorso ai politici di professione $e^{6}$ per garantire la riproduzione del ceto politico e, adottando il criterio della rappresentatività, hanno privilegiato i candidati provenienti da specifici gruppi sociali e categorie per spingere certi segmenti dell'elettorato ad identificarsi con quelli e, di conseguenza, indurli più facilmente a votarli.

Quest'ultimo aspetto appare evidente dalla tabella 5, che mostra la persistente sovraesposizione dei cosiddetti ceti economici, in particolare quelli imprenditoriali. Gli imprenditori sono stati presenti tra i candidati nei collegi maggioritari nelle elezioni del 1996 in misura significativamente superiore alle percentuali medie fatte registrare da questa categoria nelle competizioni elettorali dell'età repubblicana e anche rispetto a quelle del $1994(+2,8 \%)$, quando le candidature imprenditoriali segnarono uno straordinario incremento (Mattina 1995, 243).

La tabella 5 mostra, d'altra parte, che il peso dei dirigenti

5 I candidati insegnanti appaiono in forte decremento perché meno presenti nei collegi maggioritari delle sei regioni meridionali, ora considerate, che non facevano parte del campione di candidati selezionato nel 1994. Sulle ragioni della minore presenza dei candidati insegnanti nei collegi maggioritari si veda oltre, pp. 500-502.

6 I 233 candidati politici di professione della Tab. 5 sono distinguibili in 67 dirigenti politici nazionali e locali dei partiti; 71 politici di carriera, cioè candidati che svolgevano un'attività professionale qualificata precedente la carriera politica e che si sono presentati consecutivamente alle ultime tre elezioni politiche nazionali risultando sempre eletti o due volte su tre; 95 politici che dichiarano di svolgere un'attività professionale evidentemente non esercitata (per es. P.F. Casini si definisce «dirigente d'azienda», Clemente Mastella «giornalista») o strumentale a quella politica (soprattutto nel pubblico impiego). 
TAB. 5. Le attività professionali dei candidati (\%)

\begin{tabular}{|c|c|c|c|c|c|c|}
\hline Attività & N. & Ulivo $+\mathrm{Rc}$ & Polo & $\begin{array}{l}\text { Lega } \\
\text { nord }\end{array}$ & Totale & $\begin{array}{c}\text { Var. 1996/ } \\
1994^{* * *}\end{array}$ \\
\hline Avvocati & 168 & 7,5 & 15,6 & 3,1 & 9,7 & $-1,8$ \\
\hline Medici & 154 & 7,6 & 11,3 & 6,8 & 8,9 & $-2,5$ \\
\hline Altri professionisti & 165 & 4,9 & 10 & 17,7 & 9,6 & $+1,6$ \\
\hline Insegnanti & 136 & 11,8 & 3,7 & 8,2 & 7,9 & $-4,1$ \\
\hline Docenti universitari & 105 & 9,7 & 5 & 1,1 & 6,1 & -1 \\
\hline Magistrati & 36 & 2,7 & 2,5 & - & 2,1 & $+0,1$ \\
\hline Imprenditori & 173 & 3,2 & 14,1 & 15,5 & 10 & $+2,8$ \\
\hline Dirigenti d'azienda & 57 & 3,8 & 2,8 & 3,4 & 3,3 & $-0,2$ \\
\hline Commercianti e artigiani & i 69 & 0,3 & 3,2 & 12,7 & 4 & $+0,6$ \\
\hline Terziario privato* & 88 & 2,7 & 4,1 & 11,5 & 5,1 & $-2,3$ \\
\hline Politici di professione & 233 & 21,5 & 10,8 & 3,1 & 13,4 & $+5,4$ \\
\hline Giornalisti & 69 & 4,9 & 4,1 & 2 & 4 & $-0,6$ \\
\hline Dipendenti pubblici & 168 & 11 & 8,4 & 9,8 & 9,7 & $+3,8$ \\
\hline Sindacalisti & 41 & 4,9 & 1 & - & 2,4 & - \\
\hline Pensionati & 36 & 1,9 & 2,2 & 2,3 & 2,1 & $-0,7$ \\
\hline Altre attività & 29 & 1,6 & 1,2 & 2,8 & 1,7 & $-1,1$ \\
\hline $\begin{array}{l}\text { Tot. } \\
\text { (N.) }\end{array}$ & 1.727 & $\begin{array}{c}100 \\
(693)\end{array}$ & $\begin{array}{l}100 \\
(679)\end{array}$ & $\begin{array}{c}100 \\
(355)\end{array}$ & $\begin{array}{c}100 \\
(1.727)\end{array}$ & \\
\hline
\end{tabular}

* Comprende sia le attività autonome che i lavoratori dipendenti.

** I dati del 1994 erano relativi ai candidati presentati nei collegi uninominali di 8 regioni campione rispetto alle 20 di questo saggio.

politici e dei politici di carriera è di nuovo cresciuto dopo la rilevante flessione del 1994. Ferme restando le cautele sulla confrontabilità dei dati nelle due tornate elettorali - in quanto i primi sono stati rilevati da un campione e quelli del 1996 dall'universo dei casi - è possibile spiegare il notevole aumento di questa categoria di candidati con la decisione dei leader politici di tutti i maggiori partiti, con l'eccezione della Lega, di ricandidare i parlamentari già eletti nel 1994. Inoltre, è avvenuto il reingresso in politica (in verità limitato) di parlamentari e dirigenti che nel 1994 si erano fatti da parte. Questi ultimi hanno inciso per il $3 \%$ sul totale dei nostri candidati.

L'incremento di candidati imprenditori e politici di professione, cioè delle categorie che suggeriscono due criteri opposti di offerta della rappresentanza, l'una proveniente dalla società civile, l'altra dal circuito politico-partitico, indicano che la selezione delle candidature è stata governata, nelle elezioni del 1996, da spinte divergenti che appaiono evidenti nella stessa tabella 5 . Da essa si desume che ciascuno dei tre schieramenti in competizione ha adottato una sua specifica strategia di recluta- 
mento, puntando soprattutto su tre-quattro categorie di candidati. La loro somma dà ragione di più del $50 \%$ del totale delle candidature di ciascuno schieramento. Il Polo per le libertà ha puntato prevalentemente sui liberi professionisti e gli imprenditori $(51 \%)$ senza, però, trascurare i politici di professione. La Lega nord si è rivolta soprattutto ai ceti economici - piccoli imprenditori, commercianti ed artigiani - ed alle attività che configurano profili professionali complementari alla piccola azienda, cioè i commercialisti tra gli «altri professionisti» e gli assicuratori, i periti chimici ed industriali, i geometri, ecc. nel «terziario privato». Da questi ceti la Lega ha reclutato più del $57 \%$ dei suoi candidati. La coalizione dell'Ulivo, con il Prc, ha proposto un'offerta concentrata sui politici di professione, i dipendenti pubblici e sulle professioni del mondo della scuola (insegnanti e docenti universitari). Se a questo tipo di candidati aggiungiamo anche $\mathrm{i}$ sindacalisti, constatiamo che quasi il $59 \%$ dei candidati dell'Ulivo, con il Prc, proviene dalle categorie che per gran parte dell'esperienza repubblicana offrivano personale politico di formazione partitica.

Nel complesso è accaduto che i partiti hanno sovradimensionato, nelle proprie fila e all'interno dello schieramento di appartenenza, il peso numerico di certi tipi di candidati a svantaggio di altri, indipendentemente dal grado di rilevanza ed estensione sociale dei bacini di reclutamento trascurati. Ne è derivato un universo di candidati che non ha rispecchiato la struttura socio-politica del paese bensì quella dei ceti che per posizione o interesse sono politicamente i più attivi.

Tale risultato non deve stupire perché è quanto ci si aspetta che accada in un regime democratico nel quale i candidati designati a far parte delle assemblee elettive non possono essere selezionati su base esclusivamente funzionale o di rappresentatività sociale, bensì anche e soprattutto per le opzioni ideali e/o per i grandi interessi collettivi che intendono rappresentare. D'altra parte abbiamo prima constatato che certi tipi di candidati sono stati reclutati prevalentemente in base al criterio della rappresentatività sociale, cioè secondo una logica di selezione molto settorialistica. Cosa è, infine, accaduto? I partiti hanno prodotto un'offerta di rappresentanza non riconducibile ad alcuna opzione di ordine generale in grado di distinguere uno schieramento dall'altro? Oppure, al contrario, ciascuno schieramento è riuscito a proporre un qualche coerente messaggio politico che è deducibile dall'identità sociale e professionale dei candidati? 
A tale questione daremo più avanti una risposta. Prima di affrontare l'argomento è, però, opportuno ricostruire, per quanto possibile, il come del sovradimensionamento di certi tipi di candidati effettuato dai partiti. Tale sovradimensionamento è anche il risultato, come si dirà più avanti, delle tradizioni politiche dei diversi partiti. $\mathrm{Ma}$, più immediatamente, esso è stato realizzato, sia nel 1994 che nel 1996, sulla scorta dei condizionamenti politico-istituzionali derivanti dalla destrutturazione e ristrutturazione (ancora in corso) del nostro sistema partitico, e dai vincoli e opportunità offerte dal mercato elettorale configurato dall'adozione del sistema misto. Sull'incidenza esercitata da tali fattori nella selezione delle candidature punteremo ora la nostra attenzione. I risultati dell'analisi dovrebbero rendere meno difficile la distinzione tra i fattori congiunturali e permanenti che possono indurre i partiti e gli schieramenti ad «insistere» sullo stesso tipo di candidature.

\section{Le candidature sovradimensionate}

Per sovradimensionare e mantenere, al tempo stesso, ai candidati e allo schieramento una ragionevole possibilità di successo, le leadership partitiche hanno configurato l'incidenza delle candidature provenienti dai gruppi e categorie preferite in ragione delle caratteristiche socio-politiche dell'area di localizzazione dei collegi maggioritari. Per l'aspetto delle caratteristiche sociali si è valutata l'estensione della presenza e dell'influenza di certi ceti sociali e professioni in un determinato territorio. Per quello politico si è tenuto conto delle preferenze elettorali prevalenti in una certa area.

In termini operativi i partiti hanno particolarmente accentuato la presenza dei candidati preferiti nei collegi compresi nell'area in cui l'insediamento sociale esteso dei gruppi di riferimento e/o l'aspettativa di un risultato elettorale favorevole li incoraggiavano ad effettuare tale scelta. Per la maggior parte dei partiti del Polo l'area «amica» nella quale sovradimensionare i candidati preferiti è stata il Nord; per i partiti dell'Ulivo l'Italia centrale; per An e il Ccd-Cdu, il Sud.

Quando le caratteristiche socio-politiche dell'area sono apparse sfavorevoli al sovradimensionamento dei candidati provenienti dai gruppi preferiti, i partiti che si ritenevano in partenza svantaggiati hanno fatto ricorso ad una sorta di adattamento 
ambientale delle candidature adeguandole alle figure sociali più apprezzate nell'area e/o a categorie che sembravano offrire maggiori possibilità di rovesciare un risultato elettorale previsto come negativo. $\mathrm{Ma}$ anche in questo caso estremo i partiti non hanno mai rinunciato, per quanto è stato loro possibile, a sovrarappresentare le categorie preferite.

Nord. Nel Nord il sovradimensionamento di candidati provenienti dai ceti preferiti è stato effettuato esclusivamente da Forza Italia e dalla Lega. Tutti gli altri partiti hanno scelto, chi più chi meno, la strada dell'adattamento ambientale della loro offerta ad un contesto socio-politico non del tutto favorevole alla sovrarappresentanza di candidati provenienti dai loro gruppi di riferimento.

In particolare, Forza Italia e Lega hanno puntato sulla rappresentatività sociologica dei candidati provenienti dai ceti economici e sugli operatori del terziario privato (tab. 6). Nessun'altra formazione politica (tranne la stessa Lega, vedi oltre), in nessuna delle tre aree in cui abbiamo diviso il paese, ha sovrastimato in eguale misura quei ceti tra i propri candidati. Il sovradimensionamento è, comunque, stato effettuato con alcune significative differenze. Forza Italia ha puntato soprattutto sugli industriali titolari di imprese di media grandezza, seguiti a molta distanza dai titolari di piccole attività economiche e dagli operatori nel terziario privato. La Lega, al contrario, ha puntato prevalentemente sui candidati provenienti dalle piccole attività produttive costruendo un profilo di candidato incentrato sul piccolo produttore e sulle attività che gli ruotano attorno.

La scelta di Forza Italia e della Lega di puntare sulla rappresentatività sociologica dei candidati provenienti dai ceti economici aveva una chiara valenza politica, ma era realizzabile nella misura mostrata dalla tabella 6 solo al Nord, dove la struttura sociale dell'area consentiva un'esposizione di quelle dimensioni. Di quella struttura sociale, e della recente «mobilitazione politica» dei ceti produttivi del Nord, hanno tenuto conto anche i partiti di sinistra che per tradizione non hanno mai offerto uno spazio significativo a tali ceti nelle proprie liste. Se, infatti, consideriamo la strategia dell'offerta di rappresentanza del Pds, notiamo che esso ha selezionato le candidature con molta maggiore attenzione alla società civile di quanto non abbia fatto, come vedremo tra breve, al Centro, dove ha mirato a sovradimensionare la presenza dei candidati provenienti dalle categorie 


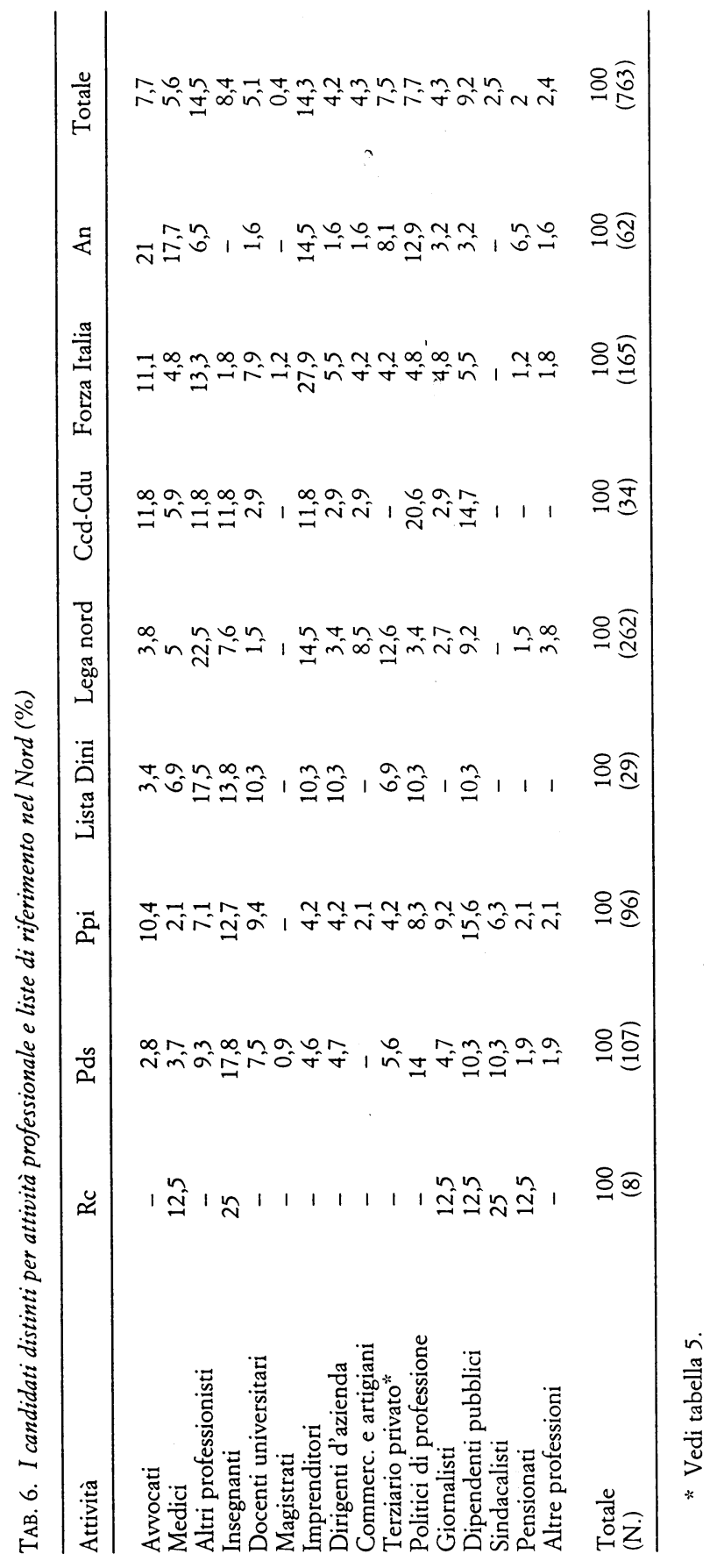


tradizionalmente preferite. Nelle zone più industrializzate del paese il Pds ha dovuto tener conto dell'esistenza di un esteso rifiuto contro la partitocrazia e di aspettative di voto sfavorevoli per l'Ulivo. Perciò, il Pds ha notevolmente ridotto il peso dei candidati politici di professione e ha accentuato - all'interno delle categorie di riferimento - quello degli insegnanti, dei dipendenti pubblici e dei sindacalisti. Naturalmente anche tali candidature contenevano un alto tasso di «partiticità». Ma esso appariva meno evidente, perché diluito, ed in diversi casi effettivamente dissolto, nel radicamento associazionistico, comunitario o settoriale, di quelle candidature.

Nonostante il carattere ambivalente delle sue candidature nel settore pubblico il Pds ha, comunque, compiuto lo sforzo di presentare candidati che fossero su una lunghezza d'onda sintonica con le caratteristiche socio-politiche del Nord e, dunque, connotati da una dimensione più societaria e meno partitica. In tal senso si spiegano anche le candidature di esponenti dei ceti economici, praticamente assenti nelle altre due aree del paese. Nel complesso le candidature del Pds nel Nord sono passate attraverso il filtro di un adattamento ambientale che ha attenuato sensibilmente, pur mantenendolo, il sovradimensionamento delle categorie professionali preferite.

Nella stessa direzione si è mosso il Ppi che ha, però, presentato un'offerta ancora più differenziata per gruppi e categorie riducendo più di quanto non abbia fatto il $\mathrm{Pds}$ il peso dei politici di professione e accentuando quello dei liberi professionisti. $\mathrm{Ma}$ anche nel Nord le categorie preferite sono state, comunque, sovradimensionante. Il 37,7\% dei candidati del Ppi proveniva, infatti, dai dipendenti pubblici e dal mondo della scuola. In particolare, il peso dei candidati insegnanti si può spiegare con il tentativo, già avviato dal Ppi di Martinazzoli, di fronteggiare il collasso della subcultura cattolica nel Triveneto e l'arretramento nelle aree di industrializzazione tradizionale con il richiamo ai valori dell'associazionismo cattolico (si veda la tab. 10), coltivato sia nelle attività di volontariato che dentro la scuola.

Anche il Ccd-Cdu al Nord si è adattato ad un contesto dominato dalla «mobilitazione politica» dei ceti economici. Perciò le due formazioni hanno presentato candidati provenienti da quei ceti senza, però, arrivare a far prevalere nella selezione il criterio della rappresentatività sociologica perché l'aspettativa di voto favorevole ai partiti del Polo ha indotto la leadership del Ccd-Cdu a concentrarsi soprattutto nella ricerca di collegi sicu- 
ri (poi rivelatisi tali solo in parte) per i suoi dirigenti politici nazionali e locali (quest'ultimi rappresentati soprattutto nella categoria dei dipendenti pubblici). In ciò le due compagini di ispirazione cattolica hanno confermato uno dei tratti caratteristici della loro offerta di rappresentanza, che appare evidente soprattutto nel Sud. Esse si qualificano, cioè, come formazioni prevalentemente costituite da politici di professione ex-democristiani, interessati a trovare un'opportunità elettorale che li mantenesse (o li introducesse) all'interno del circuito parlamentare.

Considerazioni in parte analoghe si possono fare per i candidati di An, che ha mostrato di volersi adattare al contesto socio-politico più di quanto non abbia fatto il Ccd-Cdu proponendo una significativa offerta di candidati imprenditori e di operatori del terziario privato. Ma l'adattamento è stato parziale. Come tutti gli altri partiti politici, anche An ha mirato soprattutto a sovradimensionare $i$ ceti e le categorie preferite; in questo caso gli esponenti delle libere professioni tradizionali (in una percentuale pari al $38,7 \%$ di tutti i suoi candidati) e i politici di professione, cioè i suoi dirigenti periferici da anni relegati all'opposizione negli Enti locali ed intermedi.

Centro. Forza Italia ha cercato di sovradimensionare i ceti preferiti anche nelle altre due aree del paese, ma vi è riuscita solo in parte. Nelle regioni dell'Italia centrale la formazione guidata da Berlusconi ha dato per scontata la vittoria dello schieramento avversario. Perciò Forza Italia non ha trascurato $\mathrm{i}$ candidati imprenditori e i commercianti e gli artigiani, che appaiono nelle sue liste in misura molto più rilevante di quanto non avvenga tra $i$ candidati dei partiti appartenenti allo schieramento dell'Ulivo ${ }^{7}$. Ma, nel tentativo di sovvertire un pronostico sfavorevole, Forza Italia ha puntato soprattutto su candidature che proponessero figure sociali molto visibili e presumibilmente radicate nel territorio quali i grandi professionisti ${ }^{8}$. Né si è lasciata sfuggire l'opportunità di riproporre il suo messaggio antipartitico evitando di presentare candidati politici di professio-

7 L'apparente eccezione dei candidati imprenditori collegati al Ppi è evidentemente determinata dalla distorsione del valore percentuale calcolato su un valore assoluto (11 candidati) molto limitato.

8 Non è escluso che Forza Italia abbia avuto difficoltà a collocare tra i suoi candidati del Centro gli imprenditori perché questi ultimi hanno evitato di schierarsi politicamente contro i partiti che controllano i poteri locali e con $\mathrm{i}$ quali intrattengono relazioni d'affari. 
ne. Una strategia analoga a quella di Forza Italia hanno adottato anche il Ccd-Cdu e An nei confronti dei liberi professionisti. $\grave{\mathrm{E}}$, però, interessante notare che An ha prodotto uno sforzo significativo per coprire tutta l'area dei ceti economici con un'articolazione dell'offerta che alla fine si è rivelata meno settoriale di quella proposta da Forza Italia. In particolare, An è riuscita a essere più presente anche tra $\mathrm{i}$ commercianti, gli artigiani e gli attivi nel terziario privato, a testimonianza del fatto che si è manifestata una certa radicalizzazione delle posizioni politiche di alcuni segmenti di questi ceti che si è rivelata, come vedremo tra breve, in modo ancora più esplicito nella composizione delle candidature della Lega e, per contrasto, di quelle del Pds.

Apparentemente più lineare rispetto ai partiti del Polo appare la scelta della Lega di sottolineare decisamente, la rappresentatività sociologica dei suoi candidati che provenivano, in maggioranza, dai ceti economici. In realtà nelle aree a subcultura rossa i candidati della Lega dovevano scontare previsioni non meno sfavorevoli di quelle dei candidati del Polo. Eppure Bossi non ha esitato a presentare in misura straordinariamente sovradimensionata candidati provenienti dai ceti privilegiati dalla Lega nella convinzione che ciò avrebbe posto in evidenza alcune debolezze della strategia di reclutamento dell'Ulivo, e del Pds in particolare.

Come appare evidente dalla tabella 7 , il Centro è l'area socio-politica del paese nella quale il $\mathrm{Pds}$ ha sovradimensionato in modo particolarmente esteso la categoria dei politici di professione che, sommata a quella dei sindacalisti, dà ragione del $53,2 \%$ dei suoi candidati ${ }^{9}$. La preferenza del $\mathrm{Pds}$ per i politici di professione è stata condivisa anche dalle altre formazioni dell'Ulivo e dal Prc, che hanno chiesto ed ottenuto collegi sicuri per i loro leader nazionali.

Una strategia di selezione delle candidature centrata prevalentemente sui politici di professione era realizzabile solo nell'area a subcultura rossa, anche se l'incidenza del voto di appartenenza è ivi apparsa molto attenuata negli ultimi anni ${ }^{10}$. Il Pds ha deciso di sovradimensionare pesantemente i candidati politici di professione nella convinzione - risultata confermata - di

$9 \mathrm{Ma}$ la loro incidenza è superiore perché anche buona parte dei candidati dipendenti pubblici e insegnanti svolge un'attività lavorativa che è sempre stata, o è divenuta, strumentale a quella di dirigente politico.

10 Cfr. Trigilia (1986). 


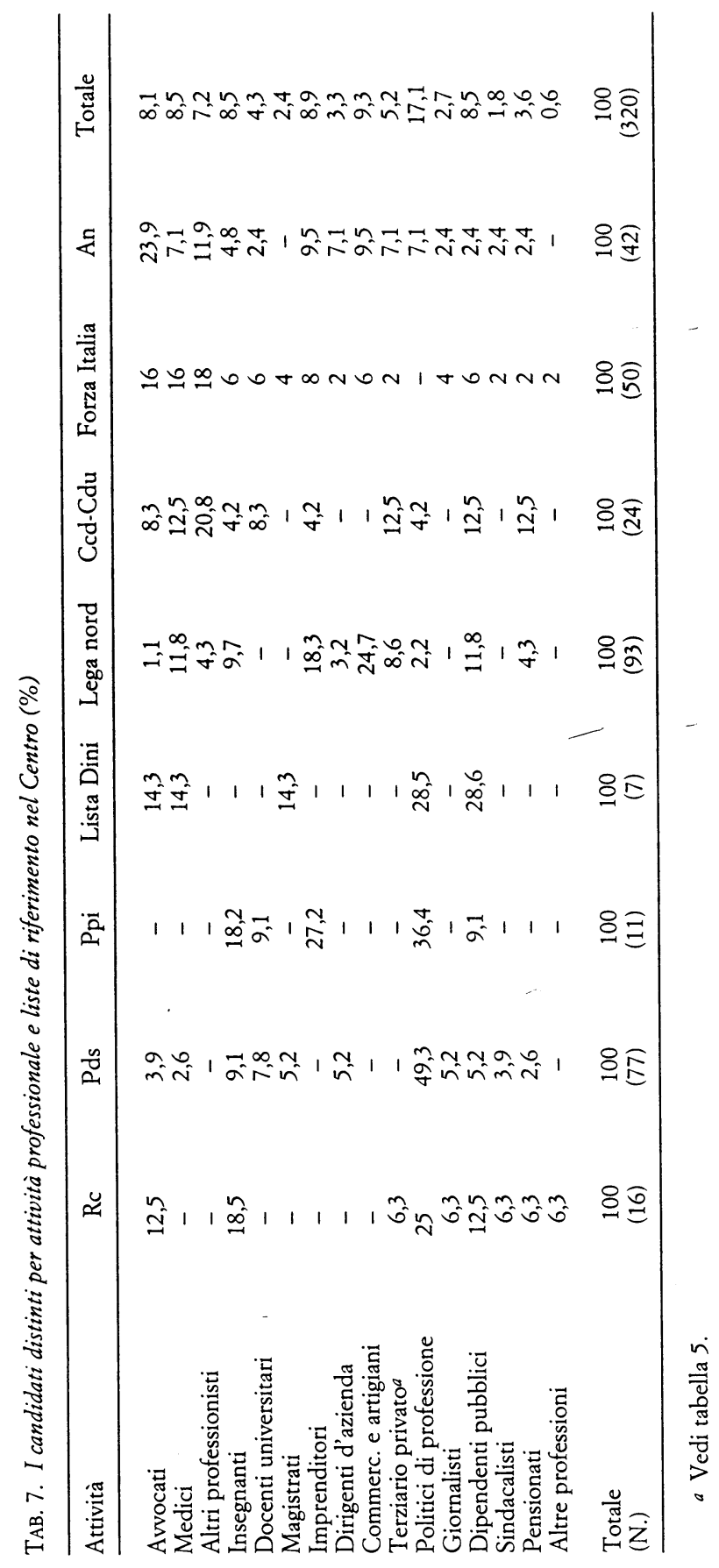


non dover temere alcun «rigetto» da parte dell'elettorato. Tale strategia ha comportato, comunque, il costo politico di trascurare la rappresentanza di altri ceti, in particolare quelli economici. La irrilevante presenza di candidati provenienti dai ceti economici collegati alle liste del Pds e dei partiti minori dell'Ulivo è evidente se confrontata con lo spazio ad essi riservato tra i candidati dei partiti del Polo; e appare addirittura sorprendente se collocata nel più ampio contesto della «mobilitazione politica» di queste categorie che è avvenuta nel Nord del paese negli ultimi anni.

A controbilanciare il disinteresse del Pds e dell'Ulivo per i ceti economici è intervenuta la Lega che nell'Italia centrale ha puntato gran parte delle sue chances di successo sui piccoli imprenditori e su commercianti ed artigiani, presentando una quota di candidati provenienti da quei ceti sociali (pari al $43 \%$ ) doppia di quella fatta registrare, per le stesse categorie, tra $\mathrm{i}$ suoi candidati nel Nord. Accentuando in misura estrema il reclutamento basato sulla rappresentatività sociologica, la Lega ha cercato di intercettare ed alimentare il malcontento (presunto o reale) di parte di quei piccoli operatori economici che comunemente si ritiene siano abbastanza protetti dai poteri locali governati dalla sinistra. Ad essi il partito guidato da Bossi ha offerto la possibilità di uscire allo scoperto candidandoli nei collegi maggioritari, in alcuni dei quali (Reggio Emilia, Parma, Piacenza, Fidenza) i candidati della Lega hanno ottenuto importanti risultati.

Si potrebbe ritenere che l'assenza nello schieramento di centro-sinistra dei rappresentanti dei ceti economici sia da attribuire principalmente alla scarsa disponibilità degli esponenti di quei ceti a candidarsi nelle fila dei partiti dell'Ulivo. Tale ipotesi appare, però, poco plausibile nell'Italia centrale dove, se i partiti di sinistra avessero voluto, avrebbero facilmente trovato candidati commercianti, artigiani o del terziario privato. Piuttosto, è più probabile che la leadership del $\mathrm{Pds}$ sia ancora convinta di poter facilmente «governare» in quell'area le richieste dei diversi gruppi sociali utilizzando la macchina politica che controlla i poteri locali e i canali di accesso alla rappresentanza politica nazionale. Il notevole numero di candidati provenienti dai settori economici che è confluito nelle fila del Polo (Forza Italia) e, soprattutto, della Lega confermano l'affinità ideologicoprogrammatica che esiste tra quei ceti e i partiti del centrodestra, ma segnalano anche l'esistenza di un qualche disagio tra 
i ceti economici sul modo in cui viene amministrato il potere in quell'area.

Sud. Nel considerare le strategie di reclutamento dei candidati adottate dai partiti nel Sud è opportuno non dimenticare che la tradizionale dipendenza della società civile dalla società politica è un dato strutturale che ha sempre influenzato trasversalmente i comportamenti di tutti i partiti politici. Come è risaputo, la debolezza della società civile deriva dalle limitate capacità autopropulsive dell'economia e della società meridionale e dalla scarsa diffusione di quell'associazionismo, laico o religioso, che nelle altre aree del paese consente una più facile emergenza dei leader di comunità o di settore che i partiti hanno cooptato in misura crescente, negli ultimi anni, dopo l'avvio delle inchieste giudiziarie a carico dei politici di professione ${ }^{11}$. Inevitabilmente, la scarsa disponibilità di leadership di formazione extra-partitica ha prodotto anche in occasione delle elezioni politiche del 1996 candidature selezionate prevalentemente attraverso $\mathrm{i}$ circuiti più tradizionali.

Nello schieramento di centro-destra Forza Italia si è distinta dagli altri due maggiori partner proponendo, anche nel Sud, il sovradimensionamento di candidature provenienti dal ceto imprenditoriale, nonostante la loro presenza e influenza sociale nel Mezzogiorno non siano paragonabili a quelle registrabili al Nord o al Centro. Minore è stata, invece, l'incidenza di commercianti ed artigiani tra i candidati di Forza Italia. Per controbilanciare tale «debolezza» nella sua offerta di rappresentanza Forza Italia è ricorsa in modo massiccio ai candidati liberi professionisti, evitando, al tempo stesso, di offrire spazio eccessivo ai candidati politici di professione o impegnati in attività strumentali a quella politica (dipendenti pubblici e insegnanti) (tab. 8). La configurazione - antipartitica e produttivistica dominante dei candidati di Forza Italia nel Sud ha, perciò, mantenuto una sua distintività rispetto ai tipi proposti sia dallo schieramento avversario che dalla sua stessa coalizione. Se esaminiamo, infatti, le caratteristiche dei candidati dei due mag.

11 Di recente è stata opportunamente segnalata un vivacità di iniziative autonome della società meridionale che smentiscono il giudizio sulla inerzia politica del Sud (cfr. Trigilia 1995). Ma quelle iniziative non offrono elementi sufficienti ad indicare l'avvio di una effettiva inversione di tendenza, se non nell'ambito delle candidature proposte per la carica di sindaco nelle città meridionali in cui si è votato con il sistema maggioritario a doppio turno. 


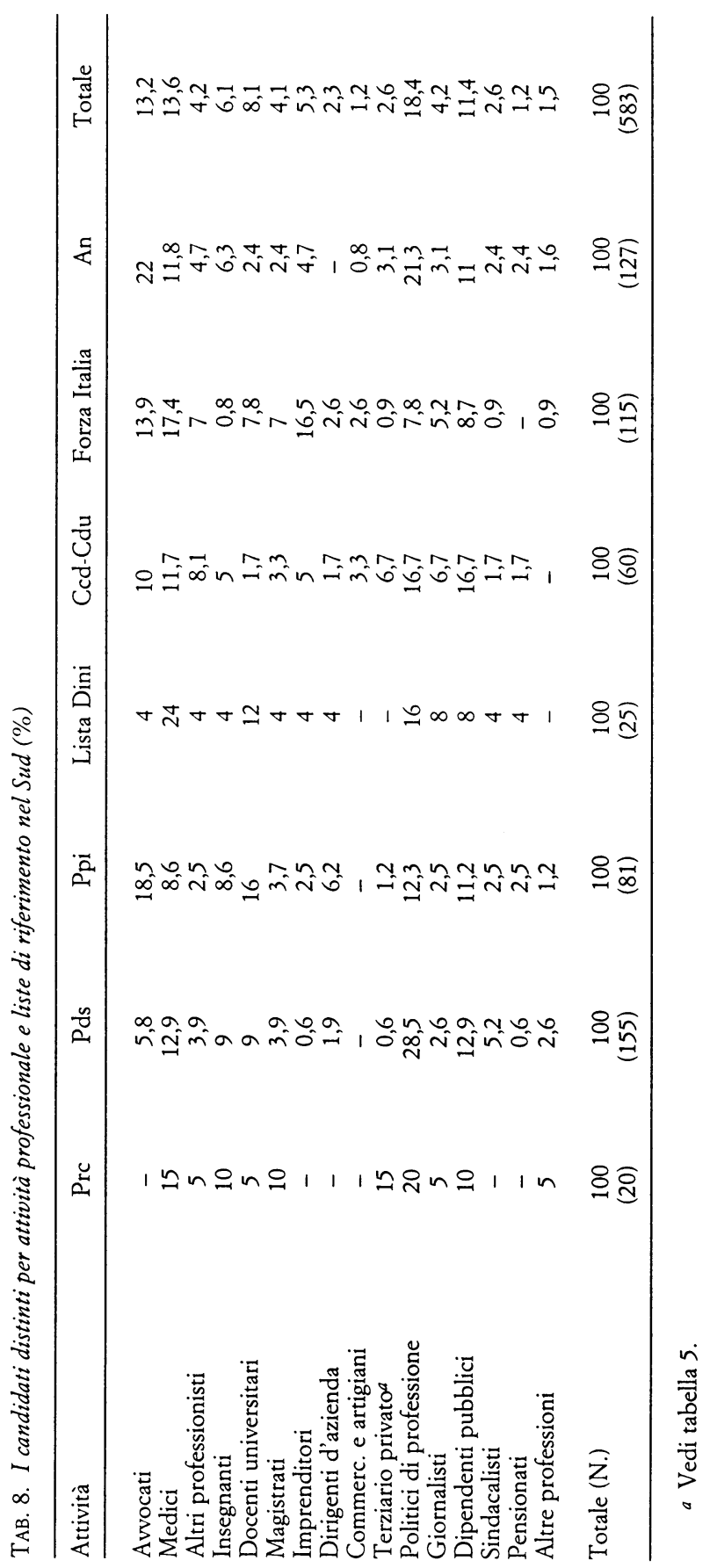


giori partner di Forza Italia nel Polo, le differenze appaiono subito evidenti.

Il Sud è stata l'area in cui Ccd-Cdu e An hanno sovradimensionato in misura estesa i loro ceti e categorie preferite con candidati nei collegi maggioritari provenienti in prevalenza dai liberi professionisti tradizionali (avvocati e medici) e dai politici di professione ${ }^{12}$. Tale combinazione rende conto di più del $51 \%$ dei candidati di An e del 38,4\% di quelli del Ccd-Cdu. Le due formazioni hanno puntato sui liberi professionisti e i politici di professione per segnalare all'elettore che i loro candidati dispongono di risorse distribuibili derivanti dai connotati notabiliari dell'attività svolta e/o dalla dimestichezza e prossimità con i centri decisionali dispensatori di tali risorse.

I due maggiori partiti dell'Ulivo hanno attuato strategie di selezione simmetriche, basate sul sovradimensionamento dei politici di professione, dei dipendenti pubblici e delle professioni del mondo della scuola - accentuando, in tale ambito, il Pds il peso dei politici e il Ppi quello dei docenti universitari. I politici di professione, insieme ai sindacalisti, danno ragione del $33,7 \%$ dei candidati del Pds (ma vedi nota 12) che conferma le sue caratteristiche di partito fortemente strutturato anche nel Mezzogiorno e la vocazione a privilegiare il peso dei dirigenti politici su qualsiasi altra categoria. Il Ppi ha accolto tra i suoi candidati un segmento significativo dei politici ex-democristiani ma ha consentito anche l'ingresso «laterale» ai rappresentanti di una delle professioni tradizionalmente più sensibili a rispondere all'appello dei partiti di centro-sinistra. E interessante notare che entrambi i partiti hanno fatto leva sui candidati insegnanti in misura significativamente più elevata di qualsiasi altra formazione politica. $\mathrm{Ma}$ l'incidenza degli insegnanti tra $\mathrm{i}$ candidati del Pds e del Ppi al Sud è, comunque, molto distante da quella registrata al Nord dove la ricchezza di iniziative associative autonome ha offerto maggiori possibilità a quei partiti di convincere all'impegno politico insegnanti inseriti in attività sociali. Lo spazio reso disponibile dalla carenza relativa di candidati insegnanti, il Pds e il Ppi lo hanno colmato facendo ricorso alle libere professioni più tradizionali, con una scelta che testimonia l'adattamento ambientale delle candidature alle caratteristiche socio-politiche dell'area.

$12 \mathrm{Ma}$ un calcolo più accurato richiederebbe la taratura della percentuale - che in quelle liste è a due cifre - dei dipendenti pubblici, cioè di un'occupazione tipicamente strumentale all'esercizio del mestiere di politico a tempo pieno (tab. 12). 
Rappresentanza e visioni politiche

L'analisi fin qui condotta sull'offerta di rappresentanza veicolata dai candidati nel maggioritario ha mostrato che le élites partitiche sovradimensionano alcuni gruppi sociali e/o categorie professionali secondo le loro preferenze.

Abbiamo, infatti, notato che i tre schieramenti hanno registrato un sensibile turnover nella composizione delle loro candidature, in particolare al Nord e al Centro (tab. 4). In più, hanno diversificato attentamente l'offerta in relazione alle specificità socio-politiche delle tre maggiori aree del paese (tabb. 6, 7, 8). Tuttavia, hanno continuato, ciascuno per la sua parte, a sovradimensionare gli stessi ceti e le medesime categorie che avevano privilegiato nelle elezioni del 1994 (tab. 5). La persistenza di tale comportamento induce a ritenere che esso abbia basi strutturali sulle quali ha inciso solo relativamente l'adozione del nuovo sistema elettorale. Non bisogna, infatti, dimenticare che ciascun partito ha un suo territorio di caccia preferito, o domain, dal quale è in grado di selezionare con relativa facilità $i$ candidati che ritiene possano meglio rappresentare gli interessi e le aspirazioni degli elettori. Abbiamo constatato che la quantità di esponenti del mondo economico che Forza Italia e Lega sono stati in grado di arruolare nelle loro fila è molto più elevata di quella fatta registrare dai partiti dell'Ulivo. Ciò perché essi hanno trovato un'oggettiva difficoltà - indipendente dalla loro disponibilità a selezionare da quel bacino sociale - a convincere esponenti dei ceti economici a candidarsi per l'Ulivo o per il Prc. Esistono, infatti, specifiche tradizioni culturali, associative e organizzative, che attengono alla formazione dell'identità sociale dei gruppi e a quella politica dei partiti, che concorrono a identificare i bacini d'utenza nei quali gli attori politici hanno maggiore facilità d'accesso nella loro ricerca di candidati e voti.

Né va dimenticato che ciascun partito tenta di mantenere i legami con le tradizioni sopra menzionate, adeguandole alle sollecitazioni del presente. E anche se il partito è di recente formazione non rinuncia a tale attitudine, richiamandosi a tradizioni altrui per dichiararsene il più genuino prosecutore (come Forza Italia); oppure si impegna nella faticosa e problematica costruzione di una tradizione propria (come la Lega). Il sovradimensionamento di certi ceti e categorie va, insomma, considerato sia una necessità che il frutto di una scelta dei partiti perché assolve all'importante funzione di favorire, in campagna elettora- 
le, l'identificabilità del partito e ribadire la sua irriducibilità agli altri. Per tale aspetto le elezioni politiche del 1996 non hanno fatto eccezione. I partiti hanno adoperato le risorse di cui disponevano per presentarsi con una forte e distinta identità. Tra queste, l'uso del sovradimensionamento di certe figure di candidati ha avuto un peso decisivo nel favorire il «riconoscimento» del partito da parte del suo potenziale elettorato. Le figure sociali o professionali dei candidati hanno evocato le visioni politiche che stanno alla base dei programmi dei partiti. Tali visioni per certi aspetti sono più facilmente ed immediatamente comunicabili dei programmi stessi attraverso la testimonianza vivente che ne danno i candidati e sono più efficaci per favorire l'identificazione dell'elettore con il partito prescelto.

Passando dai partiti agli schieramenti notiamo che, nonostante la loro eterogenea composizione, anch'essi sono stati in grado di suggerire agli elettori la visione politica che stava alla base del patto politico stipulato tra i partiti costituenti. Ciò perché si è registrata una contiguità socio-politica tra i candidati interni a ciascuno schieramento molto maggiore delle pur evidenti differenze. La coalizione dell'Ulivo, caratterizzandosi per la massiccia presenza di candidati politici di professione, insegnanti e dipendenti pubblici ha suggerito agli elettori una visione politica, del resto precisata nei programmi, centrata sul mantenimento dello stato sociale, la tutela del lavoro dipendente e, più in generale, la difesa dei ceti sociali deboli. Sull'altro versante il Polo, ed in particolare Forza Italia, con la forte presenza di candidati provenienti dalle attività imprenditoriali, dalle libere professioni e dal lavoro autonomo, ha proposto una visione politica centrata sullo stato minimo, il sostegno all'impresa e alle attività indipendenti, una legislazione poco vincolistica del mercato del lavoro. Infine la Lega, con l'esibito sovradimensionamento degli esponenti della piccola produzione e delle attività che le ruotano attorno, ha voluto dare voce ad un diffuso disagio sociale nei confronti di un sistema fiscale ritenuto iniquo e vessatorio e al tempo stesso ventilare la minaccia di una rivolta fiscale, politicamente nobilitata nella parola d'ordine del federalismo.

Le diverse visioni politiche che sono state alla base della formazione dei tre schieramenti concorrenti hanno, dunque, trovato una fedele riproduzione nella composizione delle candidature. Ciò appare evidente nella distribuzione ponderata dei candidati dei tre schieramenti sui principali quattro settori lavorativi a cui si possono ridurre le loro diverse attività professionali (fig. 1). 


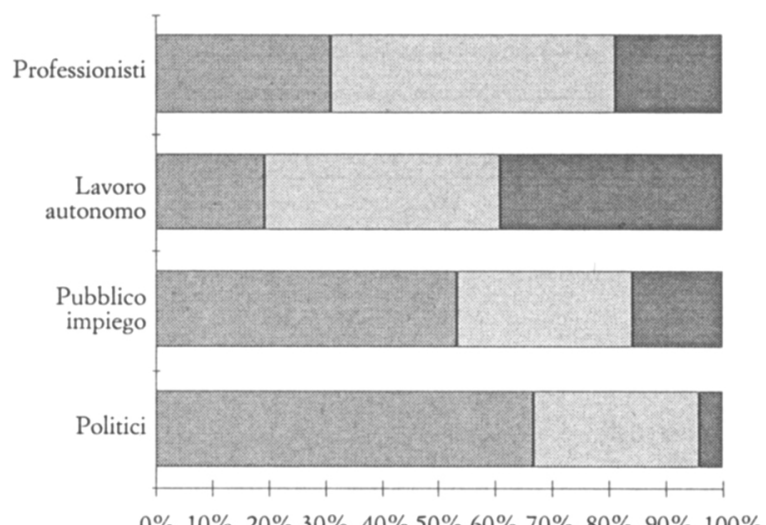

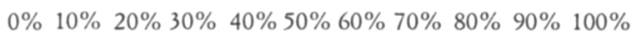

GUlivo+Prc $\square \mathrm{Ppl}$ Ln

FIG. 1. Candidati distinti per settori lavorativi omogenei (\%).

L'azione dei gruppi di interesse e delle associazioni non economiche

Nella prima parte di questo saggio abbiamo appurato che la «formazione dell'offerta» di candidature è stata fortemente controllata dalle leadership politiche nazionali. Una ulteriore conferma è data dal modesto apporto dei gruppi di interesse economici alla formazione delle candidature (tab. 9). L'impegno diretto dei dirigenti di organizzazioni imprenditoriali e sindacali ha, infatti, inciso per non più del $5,8 \%$ del totale delle candidature. Non si può, dunque, affermare che i gruppi di interesse economico si siano mobilitati in modo concorrente o parallelo ai partiti. Ciò conferma la scarsa attitudine dei gruppi di interesse all'azione politica diretta, sulle cui cause ci siamo estesamente soffermati in altra occasione (Mattina 1995, 258-262).

Più in dettaglio, emerge che i dirigenti dei sindacati dei lavoratori erano il gruppo più numeroso di candidati provenienti dalle organizzazioni di interesse, e che nella stragrande maggioranza stavano dalla parte dell'Ulivo mentre i pochi sindacalisti che si sono candidati per il Polo provenivano per la maggior parte dalla Cisnal e da sindacati autonomi di scarso peso. I dirigenti delle organizzazioni industriali e la maggioranza dei dirigenti della Confcommercio sono stati candidati, in prevalenza, dai partiti dello schieramento di centro-destra. È apparsa con- 
ТАВ. 9. I candidati dirigenti di associazioni di rappresentanza degli interessi economici. Valori assoluti

\begin{tabular}{lcccc}
\hline & Ulivo + Rc & Polo & Lega nord & Totale \\
\hline Cgil & 17 & - & - & 17 \\
Cisl & 12 & 2 & - & 14 \\
Cisnal & - & 3 & - & 3 \\
Altri sindacati & 1 & 5 & 6 & 12 \\
Sindacati & 30 & 10 & 6 & 46 \\
Confindustria & 2 & 10 & 1 & 13 \\
Confapi & - & 2 & - & 2 \\
Altre associazioni industriali & - & 5 & 4 & 9 \\
Associazioni industriali & 2 & 17 & 5 & 24 \\
Confagricoltura & - & 7 & 1 & 8 \\
Coldiretti & 1 & - & 1 & 2 \\
Confcoltivatori & 3 & - & - & 3 \\
Associazioni agricole & 4 & 7 & 2 & 13 \\
Confcommercio & 1 & 5 & 1 & 7 \\
Confesercenti & 2 & - & - & 2 \\
Altre associazioni del commercio & - & 1 & 1 & 2 \\
Associazioni del commercio & 3 & 6 & 2 & 11 \\
Confartigianato & 1 & 1 & 2 & 4 \\
Cna & - & - & 1 & 1 \\
Associazioni dell'artigianato & 1 & 1 & 3 & 5 \\
Lega delle cooperative & 4 & - & - & 4 \\
Altre associazioni cooperative & - & 1 & - & 1 \\
Totale cooperazione & 4 & 1 & - & 5 \\
Totale & 44 & 42 & 18 & 104 \\
\hline & & & & \\
\hline
\end{tabular}

fermata, inoltre, la strutturale incapacità degli artigiani di mobilitarsi politicamente attraverso $i$ loro dirigenti e a contrasto di ciò appare ancora più clamorosa la capacità della Lega di mobilitare singoli operatori di questa categoria nelle dimensioni che abbiamo in precedenza constatato. La Lega, comunque, non è riuscita a trovare simpatie tra i dirigenti delle strutture di rappresentanza economica tradizionali e quelli che ha mobilitato attraverso $\mathrm{i}$ «suoi» sindacati erano veramente pochi.

Una notazione a parte merita la collocazione dei candidati dirigenti di associazioni del settore agricolo e ciò non tanto per la dimensione del loro coinvolgimento nella partecipazione diretta alla competizione elettorale, che è stato molto modesto, quanto per la distribuzione di questa limitata presenza, che si è dislocata sull'asse sinistra-destra. Così i rappresentanti delle pic- 
TAB. 10. I candidati dirigenti di associazioni di rappresentanza di interessi non economici. Valori assoluti

\begin{tabular}{|c|c|c|c|c|}
\hline & Ulivo + Rc & Polo & Lega Nord & Totale \\
\hline Ordini professionali & 8 & 20 & - & 28 \\
\hline Arci & 5 & 1 & - & 6 \\
\hline $\begin{array}{l}\text { Acli } \\
\text { Azione cattolica } \\
\text { Altre cattoliche } \\
\text { Associazioni cattoliche }\end{array}$ & $\begin{array}{r}7 \\
10 \\
15 \\
32\end{array}$ & $\begin{array}{r}2 \\
3 \\
11 \\
16\end{array}$ & $\begin{array}{l}- \\
- \\
2 \\
2\end{array}$ & $\begin{array}{r}9 \\
13 \\
28 \\
50\end{array}$ \\
\hline Associazioni di volontariato & 18 & 14 & 3 & 35 \\
\hline $\begin{array}{l}\text { Wwf } \\
\text { Italia nostra } \\
\text { Legambiente } \\
\text { Altre ambientaliste } \\
\text { Ass. ambientaliste }\end{array}$ & $\begin{array}{r}3 \\
2 \\
10 \\
3 \\
18\end{array}$ & $\begin{array}{l}1 \\
- \\
- \\
1 \\
2\end{array}$ & $\begin{array}{l}- \\
- \\
- \\
-\end{array}$ & $\begin{array}{r}4 \\
2 \\
10 \\
4 \\
20\end{array}$ \\
\hline Associazioni sportive & 9 & 34 & 5 & 48 \\
\hline Associazioni culturali & 8 & 10 & 2 & 20 \\
\hline Ass. ex combattenti/partigiani & & 3 & - & 3 \\
\hline Ass. civiche & 3 & 3 & 1 & 7 \\
\hline Ass. femministe & 3 & - & - & 3 \\
\hline $\begin{array}{l}\text { Rotary club } \\
\text { Lions club } \\
\text { Altri club notabiliari } \\
\text { Totale club notabiliari }\end{array}$ & $\begin{array}{l}2 \\
2 \\
- \\
4\end{array}$ & $\begin{array}{r}9 \\
15 \\
4 \\
28\end{array}$ & $\begin{array}{l}1 \\
1 \\
1 \\
3\end{array}$ & $\begin{array}{r}12 \\
18 \\
5 \\
35\end{array}$ \\
\hline Altre associazioni & 18 & 8 & 5 & 31 \\
\hline Totale & 139 & 136 & 21 & 296 \\
\hline
\end{tabular}

cole e medie aziende agricole (Coldiretti e Confcoltivatori) si sono schierati prevalentemente con l'Ulivo mentre i dirigenti della Confagricoltura, in sintonia con le loro tradizioni (Mattina 1994), si sono candidati esclusivamente dalla parte del Polo.

In apparente controtendenza con la accertata centralità dei partiti nella formazione dell'offerta si presentano i dati relativi ai candidati dirigenti di associazioni non economiche (tab. 10). Costoro incidono in misura non trascurabile $(16,7 \%)$ sul totale dei candidati. Da ciò si potrebbe dedurre che sia stato attivo almeno un canale di reclutamento dei candidati, provvisto di una limitata ma definita consistenza, sottratto all'influenza dei partiti. Ma, onde evitare valutazioni semplicistiche, non bisogna di- 
menticare che i dirigenti delle associazioni non economiche raramente vantano la sola affiliazione all'organizzazione che dirigono. Più spesso sono affiliati a più di un'organizzazione. Tra queste assume particolare importanza l'affiliazione al partito politico in virtù della quale essi ottengono l'opportunità di candidarsi alle elezioni. Si pone, perciò, il problema di distinguere correttamente tra l'iniziativa indipendente del dirigente di un'associazione di entrare nella competizione elettorale e quella di un candidato dirigente che partecipa perché in realtà sollecitato in primo luogo dalla sua affiliazione partitica. Né bisogna dimenticare che per questo secondo tipo di candidato l'affiliazione partitica è spesso determinante per il suo ingresso e la successiva leadership nell'associazione non economica in quanto quest'ultima si configura come una organizzazione dipendente o ancillare al partito.

Fatte queste premesse individuiamo sinteticamente le tendenze generali che emergono dalla tabella 10 per poi suggerire qualche ipotesi sul grado di autonomia o di dipendenza dal reclutamento di tipo partitico attribuibile ai diversi dirigenti delle associazioni non economiche. I candidati dirigenti di gruppi notabiliari, ordini professionali e associazioni sportive hanno prevalso nettamente all'interno del Polo. I candidati delle associazioni cattoliche, ambientaliste e ricreative si sono schierati in prevalenza dalla parte dell'Ulivo. Si è registrata, inoltre, una presenza bilanciata nelle due coalizioni maggiori dei dirigenti delle associazioni di volontariato e culturali. Scarsa è stata la presenza di dirigenti di associazioni non economiche tra i candidati della Lega.

Alla luce delle considerazioni appena esposte possiamo ragionevolmente ipotizzare il carattere subordinato a quella partitica dell'affiliazione dei candidati dirigenti delle associazioni ambientaliste, ricreative, sportive, partigiane, femministe e culturali che si sono candidati nelle fila dell'Ulivo, in particolare nelle liste dei partiti di sinistra. Meno vincolanti ci sembrano, invece, $\mathrm{i}$ legami esistenti tra cariche direttive in ordini professionali o club notabiliari e l'eventuale affiliazione partitica. I dirigenti di questo secondo tipo di associazioni vantano anch'essi, per la maggior parte, un'affiliazione plurima, poiché la maggioranza è iscritta al partito per il quale si candida. Ma l'affiliazione partitica non ha, per così dire, «trainato» quella al club o all'ordine professionale. L'adesione a questi tipi di associazione può essere spesso preesistente o, in ogni caso, indipendente $\mathrm{da}$ 
quella al partito perché gli ordini professionali e i club notabiliari raccolgono liberi professionisti e ceti sociali che si associano per proteggere una specificità professionale e/o per sottolineare una distinzione, cioè l'appartenenza ad un'élite. Dunque, tali associazioni sono refrattarie a subordinazioni organizzative o parentele ideologiche esplicite con i partiti perché questi inevitabilmente tendono a ricomprendere specificità professionali $\mathrm{e}$ sociali in contesti più vasti. Assumiamo, cioè, che i partiti abbiano «invitato» questi dirigenti ad entrare a far parte delle loro liste elettorali perché attratti dall'eminenza della posizione sociale da essi acquisita nella comunità e dall'influenza che esercitano all'interno della categoria professionale che rappresentano.

Un caso a parte è costituito dai dirigenti delle associazioni cattoliche aderenti al Ppi che si sono candidati nell'Ulivo. La candidatura è nata da un rapporto di parentela tra l'associazione e il partito, cioè da un'affinità ideologica fondata su valori condivisi che, però, non necessariamente si traduce in un rapporto di subordinazione organizzativa al partito. Il Ppi si è affidato in modo esteso al sostegno dell'associazionismo cattolico e, quando è stato possibile, alla Cisl, che costituiscono le uniche realtà organizzative, in passato vicine alla Dc, ancora radicate nel paese. I dirigenti cattolici candidati nel Polo provenivano in prevalenza dal circuito delle associazioni collegate al Movimento popolare e a Comunione e Liberazione mentre è stata molto limitata la provenienza dalle associazioni cattoliche storiche.

\section{Le carriere politiche}

Finora ci siamo soffermati sull'offerta di rappresentanza proposta dai partiti agli elettori. I nostri dati consentono alcune considerazioni anche sul processo, più interno ai partiti, inerente la riproduzione del ceto politico. Con le elezioni del $1994 \mathrm{i}$ meccanismi tradizionali di riproduzione hanno subito una profonda modificazione a seguito dello straordinario numero di ingressi di candidati privi di esperienze politiche ${ }^{13}$. Nel 1996 la quota degli esordienti si è comprensibilmente abbassata: solo il $29,2 \%$ (pari a 515 unità) dei 1763 candidati considerati sono ri-

13 Nel 1994 abbiamo registrato un'incidenza di candidati senza precedenti esperienze politiche pari a quasi il 50\% del totale del nostro campione (Mattina 1995, 264). Ancora maggiore fu la quota degli eletti esordienti (Verzichelli 1995, 403). 
masti del tutto estranei alla politica fino al 1996. Ma se ad essi aggiungiamo i candidati parlamentari uscenti che esordirono in politica nel 1994 (135 casi), l'incidenza complessiva degli esordienti sale al 36,8\% del totale dei candidati. Ciò significa che il tasso di ricambio si è mantenuto a livelli insolitamente elevati rispetto al passato contribuendo inevitabilmente a modificare $i$ tradizionali meccanismi di selezione del personale politico, come appare evidente dai dati relativi ai candidati che nel 1996 avevano maturato almeno una esperienza politica prima o al momento della candidatura.

I dati a nostra disposizione ci dicono, infatti, che nel 1996 ben il 70,8\% dei candidati dei tre maggiori schieramenti avevano già avuto esperienze politiche. Ma di essi quasi il $60 \%$ era stato titolare esclusivamente di cariche parlamentari o elettive locali (tab. 11), cioè aveva effettuato, o avviato, un percorso inconsueto rispetto ai modelli standardizzati di carriera politica, tradizionalmente centrati sulla prevalenza della carriera esclusivamente partitica.

Nel nostro caso appare evidente la diminuita rilevanza delle carriere esclusivamente partitiche $(9,5 \%)$ a tutto vantaggio del rilievo ormai dominante che sta assumendo l'aver acquisito cariche amministrative e elettive locali. Se, infatti, tra queste consideriamo anche le cariche elettive locali detenute in combinazione con cariche partitiche, ben il 53,4\% dei nostri candidati è passato attraverso tale esperienza (tab. 11) a conferma del fatto che l'avere prestato «servizio» a livello locale è ormai divenuto un requisito indispensabile per acquisire potere e visibilità, e quindi risorse proprie per misurarsi nella difficile competizione elettorale per l'ingresso in parlamento.

La prevalenza dei due percorsi alternativi a quello partitico (l'accesso laterale al parlamento; l'esperienza elettiva locale) si riscontra nei precedenti di tutti i candidati, senza distinzione di partito. $\mathrm{Ma}$ i candidati che vantano solo una esperienza parlamentare prevalgono soprattutto nelle fila dei due partiti di sinistra, in Forza Italia e, in misura minore, in An. Ciò testimonia lo sforzo compiuto dai primi e da An di riconfermare tutti i parlamentari uscenti, e l'obiettiva necessità di una formazione priva di struttura organizzativa articolata e radicata nel territorio, quale è Forza Italia, di favorire accessi laterali al parlamento. Quest'ultimo elemento sembra smentito dall'elevato numero di candidati di Forza Italia detentori di cariche partitiche locali. In realtà costoro, nella stragrande maggioranza dei casi, sono 


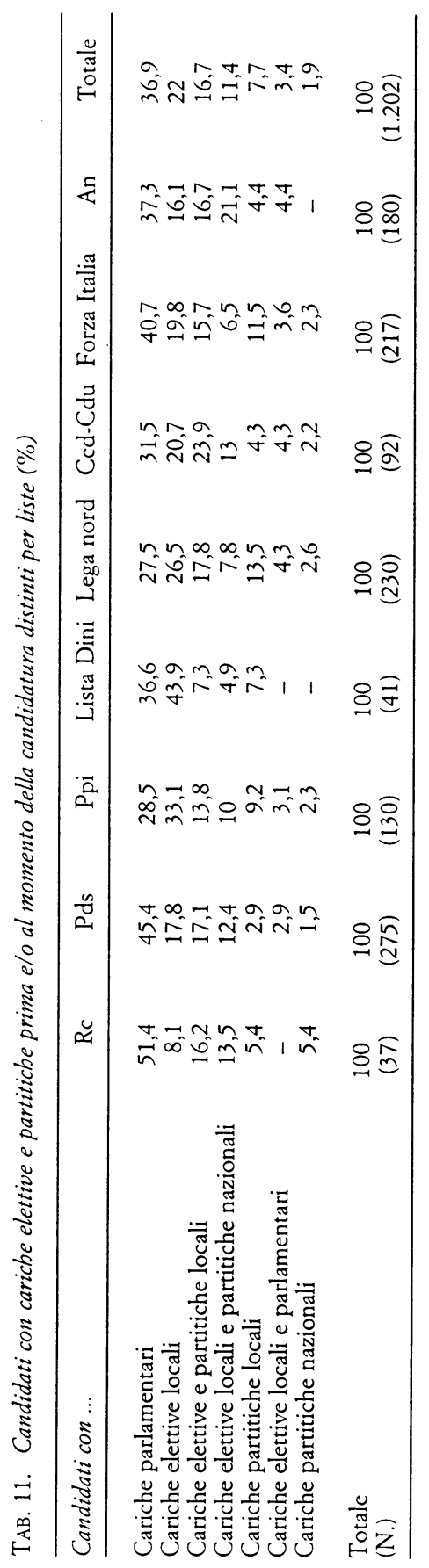


stati promotori o coordinatori locali di quei club di Forza Italia che non hanno mai acquisito lo statuto o la operatività di struttura territoriale dell'organizzazione ${ }^{14}$. I club sono stati piuttosto utilizzati dagli organi centrali come comitati elettorali da mobilitare a ridosso della scadenza elettorale a sostegno di candidati selezionati dagli organi nazionali o da quelli approvati. I candidati espressi dai club di Forza Italia provengono, in altri termi$\mathrm{ni}$, dal circuito dell'accesso laterale all'arena parlamentare e confermano la loro sostanziale estraneità al circuito formativo di tipo partitico.

A tale circuito ha, invece, attinto la Lega in misura più rilevante di qualsiasi altra formazione politica. Ma i funzionari locali della Lega vanno considerati più come militanti attivi nel territorio che come burocrati di partito. Essi rientrano, cioè, in buona misura, in quel profilo di candidato a forte connotazione localistica che costituisce la caratterizzazione dominante del candidato leghista - ben distinta dai candidati di tutti gli altri partiti - come appare evidente dalla tabella 11. L'altra formazione che, oltre la Lega, ha privilegiato, in misura superiore alla media, $\mathrm{i}$ candidati con cariche elettive e partitiche locali è stato il Ppi tra le cui fila hanno trovato ampia ospitalità i candidati provenienti dal pubblico impiego, cioè da un settore tradizionalmente «servente» $i$ partiti politici di funzionari di fatto impegnati a tempo pieno nell'attività politica.

A conferma del cambiamento avvenuto nei modelli di reclutamento del ceto politico, vale la pena sottolineare la sostanziale scomparsa tra i candidati del Pds di funzionari e dirigenti di partito, locali o nazionali. Un impianto più tradizionale della struttura di reclutamento del proprio ceto parlamentare sembra, invece, aver mantenuto An, che ha offerto notevole spazio ai dirigenti che a livello locale operano dai banchi dell'opposizione nelle assemblee comunali e regionali e ricoprono incarichi direttivi di partito a livello nazionale $(21,1 \%)$.

Ulteriori conferme ai riscontri finora emersi sui tre percorsi politici di formazione del ceto parlamentare sono desumibili dalla tabella 12 , che indica le attività professionali dei candidati con esperienze politiche. I candidati parlamentari uscenti o con incarichi parlamentari precedenti la XII legislatura, cioè quanti hanno tentato l'accesso laterale, sono stati in primo luogo i docenti universitari e i magistrati, seguiti dai politici di professione

14 Si veda sul punto Maraffi (1995). 


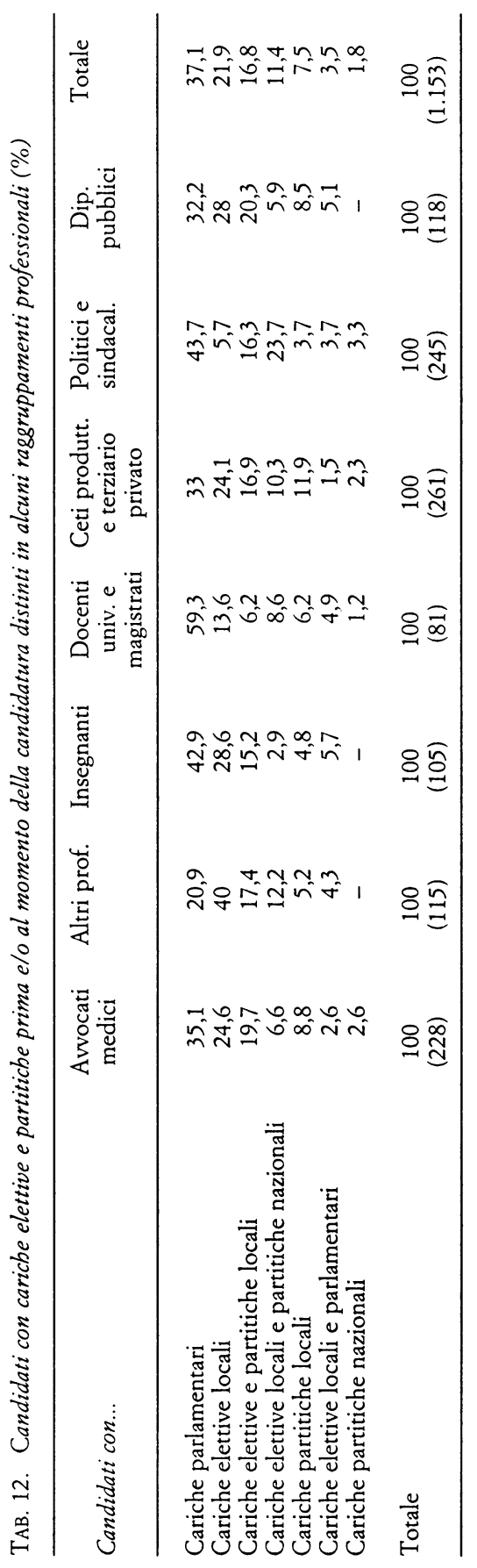


e dai sindacalisti. I candidati appartenenti al primo raggruppamento professionale sono stati scelti in virtù della loro competenza e dell'impegno scientifico o istituzionale. Perciò hanno seguito un percorso extrapartitico ed extra-amministrativo. Quanti di loro avevano già ricoperto incarichi parlamentari, se rieletti, potrebbero divenire politici di carriera. Questi ultimi sono stati compresi, nella tabella 12 , nella categoria più ampia dei politici di professione. Accanto ad essi abbiamo collocato i sindacalisti (41 casi) i quali, per via della incompatibilità tra candidatura e incarico sindacale, hanno tentato - al pari dei politici l'accesso laterale al parlamento.

Il terzo gruppo di candidati che ha manifestato una più elevata propensione all'accesso laterale è stato quello degli insegnanti; più precisamente, in questo caso, gli insegnanti con militanza prepolitica in associazioni volontarie a cui si sono rivolti nell'Italia del Nord sia i partiti di sinistra che il Ppi per presentare candidati con un radicamento territoriale da contrapporre ai più favoriti candidati del Polo. Può sorprendere, ma solo moderatamente, che i candidati avvocati e medici appartengano solo al quarto gruppo di candidati che hanno tentato l'accesso laterale. $\mathrm{Ma}$ il dato si può spiegare con la tendenza a diffondersi tra i partiti del Polo di un sistema di reclutamento del personale parlamentare fondato sulla combinazione di visibilità e prestigio sociale con l'esperienza amministrativa locale (ed anche partitica) che finora è stato dominante all'interno del MsiAn e rivolto soprattutto ai candidati avvocati ${ }^{15}$. Più prevedibile è il piazzamento in coda agli accessi laterali dei candidati provenienti dai ceti economici che è opportuno considerare insieme ai, molto distanziati, titolari di professioni complementari alle attività imprenditoriali. Costoro, come abbiamo visto in precedenza, sono stati arruolati in misura preponderante dalla Lega e perciò politicamente socializzati ad una militanza di tipo localistico. Si noti che ben il $40 \%$ degli «altri professionisti» ha ricoperto solo cariche elettive locali e che la percentuale più elevata di candidati con incarichi partitici - quasi il $12 \%$ - si registra tra $\mathrm{i}$ candidati provenienti dai ceti economici. Accanto ai rappresentanti dei ceti economici si collocano i candidati dipendenti pubblici che sono, in prevalenza, dirigenti locali di partito con cariche amministrative.

15 Questa modalità del processo di formazione del ceto parlamentare viene teorizzata da Verzichelli nell'ultima parte del suo saggio (Verzichelli 1996). 


\section{Conclusioni}

Nel corso della nostra analisi è emersa una domanda che è rimasta inespressa. Essa va ora formulata esplicitamente per darle risposta: i candidati nella quota maggioritaria sono stati importanti o, di più, decisivi ai fini del successo della coalizione dell'Ulivo sul Polo? In termini più generali, i candidati nel maggioritario in un sistema elettorale misto come il nostro, quale peso hanno ai fini del successo elettorale?

$\mathrm{Si}$ può rispondere affermando che con l'attuale sistema $\mathrm{i}$ candidati non servono a far vincere le elezioni ma, se scelti male, possono farle perdere. Chiariamo. Il sistema elettorale misto favorisce la formazione di coalizioni ma, con il mantenimento della quota proporzionale che - per di più - è prevista anche in altri tipi di elezione, impedisce il superamento della frammentazione partitica. La conseguenza è che - sul versante della quota maggioritaria - le elezioni si vincono o si perdono a seconda della qualità della strategia coalizionale adottata. Tale strategia viene attuata a livello centralizzato per dare efficacia alla logica spartitoria che presiede la formazione delle candidature promosse e sollecitate dai partiti confluenti nelle diverse coalizioni. Inevitabilmente, con tale meccanica del sistema elettorale, i candidati contano poco ai fini della vittoria perché viene di fatto ostacolato il loro radicamento territoriale mentre l'elettorato è indotto a considerare prioritariamente, ai fini della sua decisione di voto, i simboli politici esibiti dalla coalizione e la posizione da essa occupata lungo la dimensione destra-sinistra o quella centro-periferia.

Tuttavia, se i candidati vengono scelti conformemente alle caratteristiche generali esibite dalla coalizione, possono incrementare le chances di successo dello schieramento a cui appartengono. I candidati, infatti, proponendosi al giudizio degli elettori con loro caratteristiche socio-professionali e politiche, evocano dinanzi all'elettorato le visioni politiche che stanno alla base della coalizione di appartenenza, e ne sottolineano la distintività rispetto a quelle proposte dalle coalizioni concorrenti.

In sede conclusiva vale la pena di aggiungere che le diverse visioni politiche proposte dai tre schieramenti riflettono abbastanza fedelmente l'attuale insediamento sociale dei partiti che se ne sono fatti promotori. Ciò significa che appare prematura la sollecitazione di quanti invitano ad abbandonare la «lettura» del sistema partitico sulla dimensione destra-sinistra o, quanto- 
meno, a riempirla di significati diversi da quelli tradizionali della «questione sociale». Sicuramente quel tema ha perduto l'intensità con cui si manifestava in passato e accanto ad esso ne sono emersi altri che possono dislocarsi sull'asse destra-sinistra. Né è da escludere che altre dimensioni debbano essere utilizzate per integrare la «lettura» dell'articolazione del sistema partitico. La nostra analisi sulle visioni politiche dei diversi partiti ha, comunque, mostrato che tali visioni sono tuttora imperniate sul tema della distribuzione equa della ricchezza sociale e sono validamente interpretabili lungo la dimensione destra-sinistra. Basti ricordare, a titolo di esempio, che nessuna delle grandi organizzazioni di interesse ha preso ufficialmente posizione a sostegno o contro uno schieramento. Eppure, i rappresentanti del mondo del lavoro si sono collocati da una parte e quelli dell'imprenditoria industriale e agricola da un'altra. Né tale scelta è stata compiuta solo dalla «base» ma, come abbiamo visto (Tab. 9), anche le leadership sindacali si sono conformate al più tradizionale dei rituali di autocollocazione politica. Nel complesso, sono emerse Italie diverse e tra loro distanti.

L'altro tema affrontato nel nostro saggio è stato l'esame delle modalità di selezione del ceto politico. Le valutazioni a cui siamo giunti coincidono con quelle formulate nel saggio sugli eletti (Verzichelli 1996). Abbiamo, innanzitutto, registrato un significativo aumento del numero dei politici di professione, rispetto alle elezioni del 1994 e constatato la scomparsa di fatto dei politici di professione di estrazione esclusivamente partitica, con un'unica e significativa eccezione riscontrabile tra candidati della Lega. Sta, per contro, acquistando sempre più peso il percorso di formazione dei politici basato su esperienze elettive locali precedenti la candidatura. Aumentano anche i politici di carriera provenienti da professioni qualificate che i partiti reclutano per presidiare le arene decisionali in cui vengono deliberate le politiche pubbliche.

Permane, infine, una notevole disomogeneità nel sistema di reclutamento del ceto politico, opportunamente sottolineata nel saggio sugli eletti (Verzichelli 1996). Ne è causa, a nostro avviso, la permanente sovraesposizione dei ceti economici tra i candidati nella quota maggioritaria, che non ha riscontri nella storia elettorale del paese e per tale ragione va considerata come un sintomo - tra gli altri - del persistente stato di transizione incompiuta del nostro sistema politico. 
Riferimenti bibliografici

Bartolini, S. e R. D'Alimonte (a cura di) (1995a), Maggioritario ma non troppo. Le elezioni politiche del 1994, Bologna, Il Mulino.

- (1995b), La competizione maggioritaria: le origini elettorali del parlamento diviso, in Bartolini e D'Alimonte (1995a), pp. 317-372.

Censis (1994), Vecchi politici o nuovi notabili. Radiografia dei candida$t i$, Roma.

Cotta, M. (1976), Classe politica e parlamento in Italia: 1946-1976, Bologna, Il Mulino.

Diamanti, I. e R. Mannheimer (a cura di) (1994), Milano a Roma. Guida all'Italia elettorale del 1994, Roma, Donzelli.

Di Virgilio, A. (1996), Le alleanze elettorali. Identità partitiche e logiche coalizionali, in «Rivista Italiana di Scienza Politica», questo numero.

Maraffi, M. (1995), Forza Italia, in G. Pasquino (1995b), pp. 252-254.

Massari, O. (1995), La selezione dei candidati, in G. Pasquino (1995a), pp. 21-48.

Mastropaolo, A. (1993), Il ceto politico. Teoria e pratiche, Roma, La Nuova Italia.

Mattina L. (1994), Gli attori economici. Vincitori e vinti, in Diamanti e Mannheimer, pp. 151-158.

- (1995), I candidati, in Bartolini e D'Alimonte (1995a), pp. 233. 270.

Pasquino, G. (a cura di) (1995a), L'alternanza inattesa. Le elezioni del 27 marzo 1994 e le loro conseguenze, Soveria Mannelli, Rubbettino.

Pasquino, G. (a cura di) (1995b), La politica italiana. Dizionario critico 1945-1995, Roma-Bari, Laterza.

Trigilia, C. (1986), Grandi partiti e piccole imprese, Bologna, Il Mulino.

- (a cura di) (1995), Cultura e sviluppo. L'associazionismo nel Mezzogiorno, Catanzaro, Meridiana Libri.

Verzichelli, L. (1995), Gli eletti, in Bartolini e D'Alimonte (1995a), pp. 401-425.

- (1996), La classe politica della transizione, in «Rivista Italiana di Scienza Politica», questo numero. 